\title{
Anatomía del Hígado de la Rata Wistar (Rattus norvegicus)
}

\author{
Anatomy of the Liver in Wistar Rat (Rattus norvegicus)
}

\author{
Richard Möller Bredo \& Noelia Vazquez Odo
}

\begin{abstract}
MÖLLER, R. \& VAZQUEZ, N. Anatomía del hígado de la rata Wistar (Rattus norvegicus). Int. J. Morphol., 29(1):76-79, 2011.
RESUMEN: La rata de laboratorio (Rattus norvegicus albinus) ha sido usada como modelo para investigaciones médicas, biológicas y moleculares, desde hace mucho tiempo. Es interesante el hecho de que no existen descripciones detalladas de la anatomía del hígado y sus ligamentos que lo fijan a la pared. El objetivo de este trabajo es definir en forma clara y acorde a los principios de la Nomina Anatomica Veterinaria el hígado y sus medios de unión en esta especie de mamífero de laboratorio. Se utilizaron 88 ratas (Rattus norvegicus albinus) de entre 250 y 450 gramos. Fueron disecadas en fresco luego de ser eutanasiadas por sobredosis de tiopental sódico intraperitoneal. Se realizó una incisión paramediana que se extendía desde el cartílago xifoides del esternón hasta el pubis y otra sobre cada arco costal hacia dorsal. Se observó que presentaba 6 lóbulos bien definidos y no presentaba vesícula biliar. Sus medios de unión eran similares a los descritos para el perro a excepción de la presencia del ligamento hepatoomental.
\end{abstract}

PALABRAS CLAVE: Anatomía; Morfología; Lóbulos hepáticos; Peritoneo.

\section{INTRODUCCIÓN}

La rata de laboratorio (Rattus norvegicus albinus) ha sido usada como modelo para investigaciones médicas, biológicas y de diversa índole desde hace mucho tiempo. Es la especie más empleada en investigación biomédica teniendo en cuenta el volumen de información existente acerca de ella. En el año 1999 2,6 millones de ratas (27\% de los animales usados) fueron utilizados en la Comunidad Europea. Es interesante el hecho de que no existen descripciones detalladas de la anatomía del hígado y sus ligamentos que lo fijan a la pared pese a que existen varios artículos y libros sobre su morfología (Chiasson, 1969; Greene, 1963; Langer, 2002). Gershbein \& Elias (1954) describieron la anatomía del hígado pero la terminología no se ajusta a la empleada por la Nomina Anatomica Veterinaria. Lo mismo sucede para la descripción de las demás partes del aparato digestivo (Ofusori et al., 2008a; Ofusori et al., 2008b; Robert, 1971; Sharp \& La Regina, 1998). El objetivo de nuestro trabajo es describir el hígado y sus medios de unión en la rata de laboratorio, acorde a los principios de la Nomina Anatomica Veterinaria (NAV) (2005).

\section{MATERIAL Y MÉTODO}

Se utilizaron 88 ratas (Rattus norvegicus albinus) de entre 250 y 450 gramos de peso criadas en condiciones acordes con el Manual sobre el cuidado y uso de los animales de experimentación (Canadian Council on Animal Care, 1998). Fueron disecadas en fresco luego de ser eutanasiadas por sobredosis de tiopental sódico intraperitoneal. Se abordó el abdomen por una línea paramediana y mediante dos incisiones sobre el arco costal hacia dorsal se observaron las vísceras del aparato digestivo.

\section{RESULTADOS}

El hígado se ubicaba sobre la cara caudal del diafragma extendiéndose a ambos lados del plano mediano desde el arco costal derecho hasta el izquierdo. Presentaba para su estudio las caras: parietal (craneal) de forma convexa relacionada al músculo diafragma y una cara visceral (caudal) cóncava relacionada al estómago y parte craneal del duodeno. Se observó que estaba dividido en los lóbulos derechos (lateral y medial), izquierdos (lateral y medial), cuadrado (muy pequeño) y caudado el cual presenta los procesos caudado y papilar (subdividido en parte dorsal y ventral). El proceso caudado tomaba contacto con el riñón derecho y el proceso papilar acabalgaba sobre la curvatura menor del estómago. No presentaba vesícula biliar. 


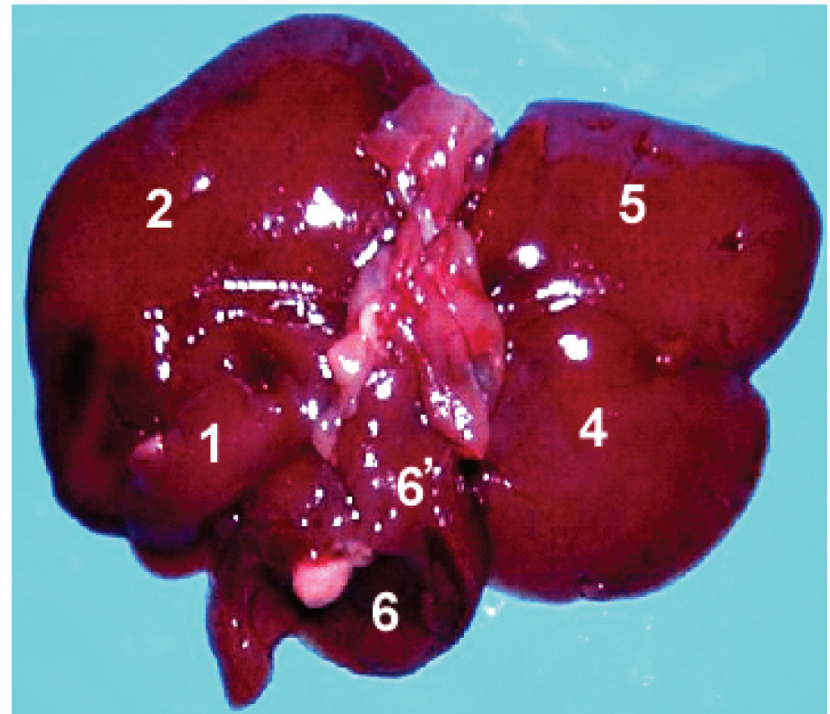

Fig. 1. Cara visceral del hígado. 1. Lóbulo lateral derecho; 2. Lóbulo medial derecho; 4. Lóbulo lateral izquierdo; 5. Lóbulo medial izquierdo; 6. Proceso caudado del lóbulo caudado.

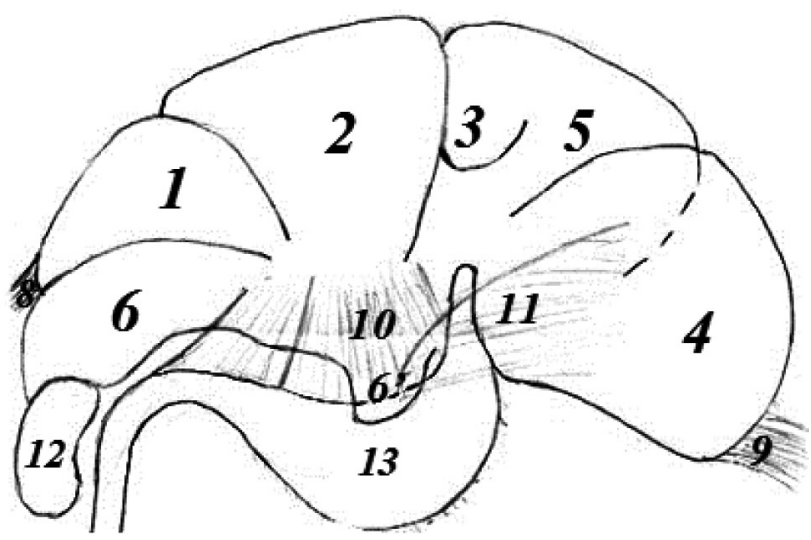

Fig. 3. Cara visceral del hígado. 1. Lóbulo lateral derecho; 2. Lóbulo medial derecho; 3 . Lóbulo cuadrado; 4. Lóbulo lateral izquierdo; 5. lóbulo medial izquierdo; 6. Proceso caudado del lóbulo caudado; 6'. Proceso papilar del lóbulo caudado; 8. Ligamento triangular derecho; 9. Ligamento triangular izquierdo; 10. Omento menor; 11. Ligamento hepatoomental; 12. Riñón derecho; 13. Estómago.

Se observó un ligamento falciforme, sin grasa, transparente que se extendía hacia craneal desde la cara parietal del hígado hasta el diafragma sobre el plano mediano llegando hasta el apéndice xifoides. En algunos animales continuaba hasta el ombligo con grasa dependiendo del estado corporal del animal; es decir que el ligamento falciforme completo era inconstante. Hacia dorsal se observó el ligamento triangular derecho que se extendía desde la pared abdominal dorsalmente hasta el lóbulo lateral derecho. Sobre el lado izquierdo se apreciaba el ligamento triangular izquierdo también proveniente desde la pared dorsal, bien

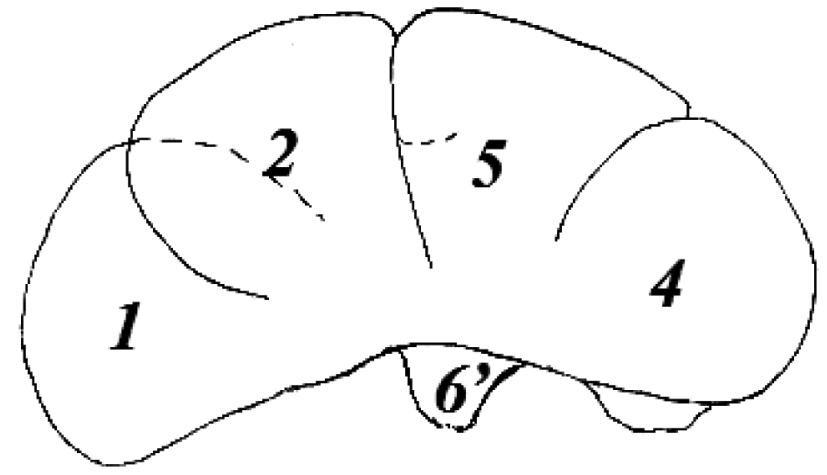

Fig. 2. Cara parietal del hígado. 1. Lóbulo lateral derecho; 2. Lóbulo medial derecho; 4. Lóbulo lateral izquierdo; 5 . Lóbulo medial izquierdo; 6'. Proceso papilar del lóbulo caudado.

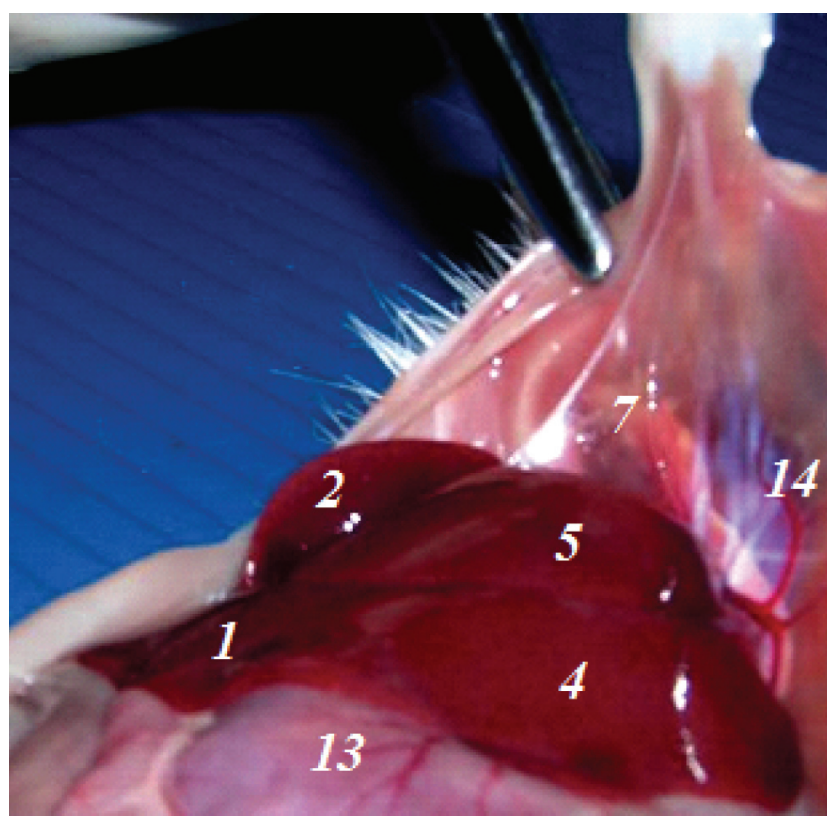

Fig. 4. Cara parietal del hígado. 1. Lóbulo lateral derecho; 2. Lóbulo medial derecho; 4. Lóbulo lateral izquierdo; 5. Lóbulo medial izquierdo; 13. Estómago, 14. Diafragma.

desarrollado, fijado al lóbulo lateral izquierdo del hígado. El ligamento hepatorrenal se extendía desde el proceso caudado del lóbulo caudado hasta una ubicación dorsal de la pared abdominal, medialmente al riñón derecho insertándose sobre la hoja derecha del mesoduodeno o sobre la hoja derecha del mesocolon descendente. El ligamento coronario era siempre relativamente pequeño. Por la cara visceral, sobre el hilio, se insertaba el omento menor que presentaba una parte izquierda fijada a la curvatura menor del estómago a la cual denominamos ligamento hepatogastrico y una parte derecha fijada al duodeno y que denominamos ligamento hepatoduodenal; el cual presentaba grasa. Por esta misma cara se encontraba también un ligamento peritoneal 
que se extendía desde el lóbulo lateral izquierdo hasta la hoja superficial del omento menor que terminaba sobre el borde de la curvatura menor del estómago al cual denominamos ligamento hepatoomental.

\section{DISCUSIÓN}

Aunque la rata de laboratorio (género Rattus) es muy utilizada para la investigación en diversas áreas del conocimiento, la anatomía del hígado no ha sido motivo de atención y no hemos encontrado bibliografía que describa las partes del órgano según los principios de la Nómina Anatómica Veterinaria.

Gershbein \& Elia describieron al hígado de la rata sin ajustar la descripción a la nomenclatura y principios de la Nómina Anatómica Veterinaria.

En esta especie, hemos observado que el hígado presenta una lobulación semejante al perro (Evans, 1993) tanto en ubicación como en lobulación a excepción del proceso papilar que se encuentra dividido en la rata. Por lo tanto, las relaciones topográficas del órgano eran similares a las observadas en el perro.

Al igual que en los caballos (Shaller, 1996), en la rata no está presente la vesícula biliar. Con respecto a los ligamentos que sostienen al hígado, se ha observado una similitud importante con los mamíferos domésticos constatando la presencia en la rata de los ligamentos descritos para estas especies. No obstante, se observó y se destaca la presencia del ligamento hepatoomental descrito por nosotros según los resultados observados. Como conclusión podemos afirmar que el hígado de la rata (Rattus) es similar a los mamíferos domésticos con características que lo asemejan a los herbívoros y a los carnívoros.

\section{AGRADECIMIENTOS}

Expresamos nuestro más profundo agradecimiento al Prof. Dr. Álvaro Freire por proveernos de los animales, a nuestras compañeras de área Bachilleres Diana Teliz y Virginia Méndez por su invalorable colaboración en las tareas de preparación y disección.

\section{MÖLLER, R. \& VAZQUEZ, N. Anatomy of the liver in Wistar rat (Rattus norvegicus). Int. J. Morphol., 29(1):76-79, 2011}

SUMMARY: The laboratory rat (Rattus norvegicus albinus) has long been used as a model for medical, biological and molecular research. It is interesting that there are no detailed descriptions of the gross anatomy of the liver and ligaments that attach it to the abdominal wall. The aim of this study is to define clearly and according to the principles of the Nomina Anatomica Veterinaria, the liver and the form of attachment in this species of laboratory mammal. Eighty eight rats (Rattus novergicus albinus) were used with a weight between 250 and 450 grams. They were freshly dissected after being euthanized by an overdose of thiopental sodium given intraperitoneally. An incision from the xiphoid cartilage up to the pubic region was made and another on each costal arch toward the dorsal. Six well defined lobes were observed; gallbladder was not present. Forms of attachment were similar to those described for the dog except for the presence of the hepatoomental ligament.

KEY WORDS: Anatomy; Morphology; Liver's lobes; Peritoneum.

\section{REFERENCIAS BIBLIOGRÁFICAS}

Canadian Council on Animal Care. Manual sobre el cuidado y uso de los animales de experimentación. Ottawa, Bradda Printing Services, 1998.

Chiasson, R. Laboratory anatomy of the white rat. $2^{\mathrm{a}}$. ed. Arizona, Wm. C. Brown, 1969. p.81.

Evans, H. Miller's Anatomy of the dog. Third Ed. New York, Saunders Company, 1993.

Gershbein, L. L. \& Elias, H. Observations on the anatomy of the rat liver. Anat. Rec., 120:85-98, 1954.
Greene, E. Anatomy of the rat. New York, Hafner Publishing Company, 1963.

Langer, P. The digestive tract and life history of small mammals. Mammal Rev., 32:107-31, 2002.

Nomina Anatomica Veterinaria (NAV). Fifth ed. International Committee on Veterinary Gross Anatomical Nomenclature (I.C.V.G.A.N.) and authorized by the General Assembly of the World Association of Veterinary Anatomists (W.A.V.A.). Knoxville, TN (U.S.A.) 2003. Published by the Editorial Committee. 
Hannover, Columbia, Gent, Sapporo. 2005. http:// www.wava-amav.org/Downloads/nav_2005.pdf

Ofusori, D. \& Caxton-Martins, E. A Comparative Histomorphometric Study of the Stomach of Rat (Rattus norvegicus), Bat (Eidolon helvum) and Pangolin (Manis tricuspis) in Relation to Diet. Int. J. Morphol., 26:66974,2008 a.

Ofusori, D.; Caxton-Martins, E.; Komolafe, O.; Oluyemi, K.; Adeeyo, O.; Ajayi, S., et al. Comparative Study of the Ileum in Rat (Rattus norvegicus), Bat (Eidolon helvum) and Pangolin (Manis tricuspis) as Investigated Using Histological Method. Int. J. Morphol., 26:13741, 2008b.

Robert, A. Proposed terminology for the anatomy of the rat stomach. Gastroenterology, 60:344-5, 1971.

Shaller, O. Nomenclatura anatómica veterinaria ilustrada. Zaragoza, Ed. Acribia, 1996.

Sharp, P. \& La Regina, M. The Laboratory rat. Ed. CRC Press, 1998.
Dirección para correspondencia:

Dr. Richard Möller

Área de Anatomía

Departamento de Morfología y Desarrollo

Facultad de Veterinaria

Universidad de la República

Lasplaces 1550

C.P. 11600

Montevideo

URUGUAY

Email: anatomia.uy@gmail.com

Recibido : 29-09-2010

Aceptado: 18-12-2010 